Anaesthesist 2010 • 59:614-620 DOI 10.1007/s00101-010-1733-7

Online publiziert: 25. Juni 2010

(c) Springer-Verlag 2010

A. Schmitz - B. Salgo - M. Weiss - C.M. Dillier - A. Frotzler · A.C. Gerber

Anästhesieabteilung, Universitäts-Kinderkliniken Zürich

\title{
Intrathekale Opioidmedikation zur perioperativen Analgesie bei schwer behinderten Kindern mit Wirbelsäulenoperationen
}

\section{Hintergrund und Fragestellung}

Wirbelsäulenchirurgische Operationen sind mit starken postoperativen Schmerzzuständen assoziiert. Für die Behandlung dieser Schmerzen stehen bei sonst gesunden Kindern mit idiopathischer Skoliose verschiedene Konzepte zur Verfügung, die zur Zufriedenheit der Patienten führen können. Werden jedoch bei kranken Kindern mit stark eingeschränkten physiologischen Reserven wirbelsäulenchirurgische Eingriffe durchgeführt, so müssen einige Besonderheiten berücksichtigt werden $[3,8]$.
Aufgrund derzeit verfügbarer Daten scheinen intrathekal verabreichte Opioide bei Schwerbehinderten spezifische Vorteile zu haben $[7,9,14,18]$ : Sie vermindern den Bedarf an i.v.-Opioid-Gaben während des Eingriffs und führen in vielen Fällen zu einer lang anhaltenden und wirkungsvollen postoperativen Analgesie. Zudem verbessern intrathekal verabreichte Opioide die hämodynamische Stabilität und reduzieren den Blutverlust $[3,5,6,8]$. Sogar stark eingeschränkte Kinder konnten nach der intrathekalen Opioidapplikation früh extubiert werden [3]. Die frühe Extubation mit einer adäquaten Schmerzbehand-

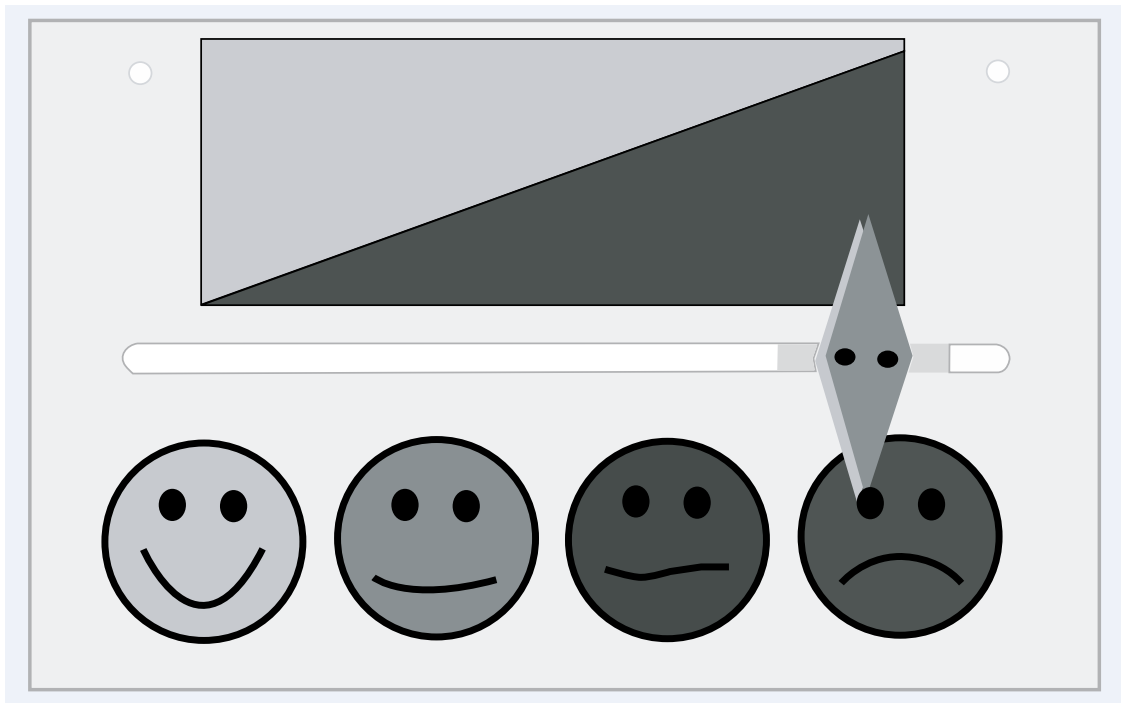

Abb. $1 \Delta$ Visuelle Analogskala (VAS) für Kinder ab 3,5 bis 6 Jahren mit farbigen Gesichtern (lachender Smiley gelb im Original, 2. Smiley von links orange im Original, 2. Smiley von rechts violett im Original, Smiley mit traurigem Gesicht rot im Original). Die Smiley-Tafel verfügt auf der Rückseite über eine Zahlenskala von 0-10 lung, die nicht die Kooperation des Patienten erfordert, und eine rasche Rückverlegung auf die Normalabteilung sind gerade bei schwer behinderten Kindern äußerst erstrebenswert. Diese Kinder haben in der Regel geringe respiratorische Reserven $[17,22]$. Sie reagieren sehr empfindlich auf die Gabe systemischer Opioide, sind häufig nicht kooperationsfähig und können ihre Schmerzen nur in beschränktem Maß ausdrücken $[15,19]$.

Das Ziel der vorliegenden Untersuchung war es, die Anwendbarkeit von intrathekal verabreichten Opioiden bei schwer- und schwerstbehinderten Kindern für wirbelsäulenchirurgische Eingriffe zu überprüfen.

\section{Studiendesign und Untersuchungsmethoden}

Mit Zustimmung der lokalen Ethikkommission wurden 28 aufeinanderfolgende Patienten vom Status 3 und 4 der Klassifikation der American Society of Anesthesiologists (ASA), bei denen ein wirbelsäulenchirurgischer Eingriff mit intrathekaler Opioidgabe durchgeführt wurde, untersucht. In die Studie wurden alle $\mathrm{Pa}$ tienten der ASA-Klasse 3 und 4 über einen Erfassungszeitraum von 6 Jahren aufgenommen; ansonsten gesunde Kinder und Jugendliche mit idiopathischer oder traumatisch bedingter Skoliose wurden ausgeschlossen.

Die Prämedikation der Patienten erfolgte mit Midazolam oder Flunitrazepam, anschließend wurde die Allgemeinanästhesie inhalativ mit Sevofluran oder i.v. mit Propofol eingeleitet. Nach der tra- 


$\begin{array}{llllll}\text { Tab. } 1 & \text { Alter, Gewicht und Länge mit Perzentilenverteilung } & \\ & \text { Median } & \text { Minimum } & \text { Maximum } & \text { Mittelwert } & \text { Standardabweichung } \\ \text { Alter (Jahre) } & 11,6 & 2,8 & 18,5 & 12,5 & \pm 3,9 \\ \text { Gewicht }(\mathrm{kg}) & 26,8 & 11,0 & 76,0 & 28,3 & \pm 13,9 \\ \text { Gewichtsperzentile } & 0 & 0 & 79 & 11,6 & \pm 26,6 \\ \text { Länge (cm) } & 131 & 89 & 165 & 130,7 & \pm 20,4 \\ \text { Längenperzentile } & 0 & 0 & 100 & 9,2 & \pm 21,5\end{array}$

chealen Intubation wurden je ein zentraler Venenkatheter, ein Arterienkatheter und ein Blasenkatheter eingelegt. Mithilfe einer perkutanen Spinalpunktion wurden im Bereich der unteren Lendenwirbelsäule $20 \mu \mathrm{g}$ Morphin und $1,5 \mu \mathrm{g} / \mathrm{kgKG}$ Sufentanil intrathekal injiziert. War die Lumbalpunktion nicht erfolgreich, wurde die identische Morphin- bzw. Sufentanildosis nach chirurgischer Präparation der Dura mater durch den Chirurgen intrathekal appliziert. Paracetamol wurde nach der Anästhesieeinleitung in einer Dosis von $20-40 \mathrm{mg} / \mathrm{kgKG}$ rektal verabreicht und postoperativ mit $100 \mathrm{mg} / \mathrm{kg}$ KG und Tag, verteilt auf 3 bis 4 Einzeldosen, fortgeführt. Zur Aufrechterhaltung der Allgemeinanästhesie wurde Propofol i.v. oder Sevofluran verwendet und erforderlichenfalls mit Fentanyl oder Alfentanil supplementiert. Die Beatmung erfolgte mit einem Sauerstoff-Lachgas-Gemisch, die Muskelrelaxierung wurde mit Atracurium oder Pancuronium durchgeführt. Ein Neuromonitoring mit evozierten motorischen oder sensorischen Potenzialen wurde nicht vorgenommen.

Zur Prophylaxe von postoperativer Übelkeit und Erbrechen („postoperative nausea and vomiting", PONV) wurde 20 min vor der Extubation o, $1 \mathrm{mg} / \mathrm{kg}$ KG (max. 2 mg) Tropisetron i.v. appliziert. Die Patienten wurden unmittelbar nach Beendigung des Eingriffs extubiert, wenn keine der folgenden Kontraindikationen vorlag: Hypothermie $\left(<35,5^{\circ} \mathrm{C}\right)$, Massentransfusion oder präoperativ bestehende ausgeprägte Muskelschwäche. Das Vorliegen dieser Kontraindikationen und die klinische Einschätzung durch den verantwortlichen Anästhesisten am Ende der Operation waren Entscheidungsgrundlage für eine verzögerte Extubation. Patienten, die auf der Intensivpflegestation nachbeatmet wurden, erhielten Midazolam zur Sedierung. Alle Patienten wurden postoperativ auf der pädiatrischen Intensivpflegesta- tion bis zur Verlegung auf die chirurgische Allgemeinstation überwacht.

Die anhaltende intrathekale Opioidwirkung und die Fortführung der Paracetamolmedikation bildeten die Basis der Analgesie. Zur Vermeidung von ausgeprägten Atemdepressionen, PONV und Pruritus sowie zur Erreichung einer gewissen Analgesiewirkung via $\kappa$-Rezeptoren wurde Nalbuphin in einer Dosis von ca. $0.1 \mathrm{mg} / \mathrm{kgKG}$ und $\mathrm{h}$ über $48 \mathrm{~h}$ kontinuierlich verabreicht. Bei Bedarf konnten zusätzliche Boli gegeben werden. Bei unzureichender Analgesiewirkung wurde die Schmerztherapie auf i.v.-Morphin-Gabe umgestellt. Zur Quantifizierung der analgetischen Wirkung wurde die etablierte visuelle Analogskala (VAS; 0 Abb. 1) eingesetzt, soweit die Kinder zur Kommunikation in der Lage waren. Andernfalls erfolgte eine Schmerzbeurteilung durch erfahrenes pädiatrisches Pflegepersonal und mithilfe einer Befragung der Eltern, deren Einschätzung ebenfalls anhand der VAS quantifiziert wurde. Die VAS wurde beim wachen Patienten bis zu stündlich über maximal $48 \mathrm{~h}$ erfasst; hierbei wurden die Werte arithmetisch auf ganze Zahlen von o-10 gerundet. Ein VAS >3 wurde als inadäquate Analgesie und bei Persistenz trotz Nalbuphinboli als Indikation für Morphin bewertet. Bei Auftreten von PONV erfolgten zusätzliche Gaben von Tropisetron bis maximal 3 Boli/Tag.

Im OP wurden der Bedarf an Anästhetika, die Notwendigkeit von zusätzlichen i.v.-Opiat-Gaben nach intrathekaler Injektion der Opioide und die Extubationszeiten aufgezeichnet. Für die postoperative Phase wurden die Gesamtdosis des verabreichten Nalbuphins berechnet und der Bedarf an zusätzlichem i.v.-verabreichten Morphin notiert. Des Weiteren wurden arterielle Kohlendioxidpartialdruck- $\left(\mathrm{p}_{\mathrm{a}} \mathrm{CO}_{2}\right)$-Werte aus Blutgasanalysen, Extubationszeit auf der Intensivpflegestation, allfällige Nebenwirkungen wie

\begin{tabular}{|c|c|}
\hline Diagnosen & $\mathrm{n}$ \\
\hline Zerebralparese & 9 \\
\hline Dysmorphiesyndrome & 10 \\
\hline Arthrogryposis multiplex & 4 \\
\hline Duchenne-Muskeldystrophie & 1 \\
\hline Myopathie, nicht näher spezifiziert & 1 \\
\hline Myeolomeningozele & 2 \\
\hline Spinale Muskelatrophie & 1 \\
\hline
\end{tabular}

PONV und Pruritus sowie der Zeitpunkt der Verlegung auf die Allgemeinstation erfasst. Die Auswertung der Daten erfolgte mithilfe der deskriptiven Statistik; die Ergebnisse werden als Median (Minimal- und Maximalwert) sowie arithmetischem Mittelwert $( \pm$ Standardabweichung) dargestellt.

\section{Ergebnisse}

Insgesamt wurden 28 Patienten im Alter von 2,8 bis 18,5 Jahren (Median 11,6 Jahre) mit einem Gewicht zwischen 11 und $76 \mathrm{~kg}$ (Median 26,8 kg) in die Untersuchung aufgenommen. Hiervon waren 8 Patienten weiblich, 17 männlich. Das Gewicht von je 21 der 28 Kinder lag unterhalb der 3. Gewichts- bzw. Längenperzentile (weitere Angaben zu Gewicht, Länge und Perzentilen: • Tab. 1). Während der Prämedikation wurden 26 Patienten als ASAStatus 3 und 2 Patienten als ASA-Status 4 klassifiziert. Die Grunderkrankungen der Patienten sind in $\bullet$ Tab. 2 zusammengefasst. Der mentale Zustand wurde präoperativ bei 9 Patienten als altersentsprechend normal, bei 5 Patienten als leicht retardiert und bei 14 Patienten als stark retardiert beurteilt. Eine perkutane Gastrostomie infolge einer Schluckstörung lag bei 5 Patienten vor.

Die Anästhesie wurde inhalativ bei 15 Patienten und i.v. bei 13 Patienten eingeleitet. Eine transkutane Lumbalpunktion konnte bei 22 Patienten erfolgreich durchgeführt werden. Bei den übrigen 6 Patienten führte der Chirurg die intrathekale Opioidinjektion nach chirurgischer Freilegung der Dura mater durch. Es wurden $20,2 \mu \mathrm{g} / \mathrm{kgKG}(13-25 \mu \mathrm{g} / \mathrm{kg}$ KG) Morphin und 1,53 $\mu \mathrm{g} / \mathrm{kgKG}\left(0,9^{-}\right.$ $2,2 \mu \mathrm{g} / \mathrm{kgKG}$ ) Sufentanil intrathekal appliziert. Die ausreichende Analgesie bis zur 


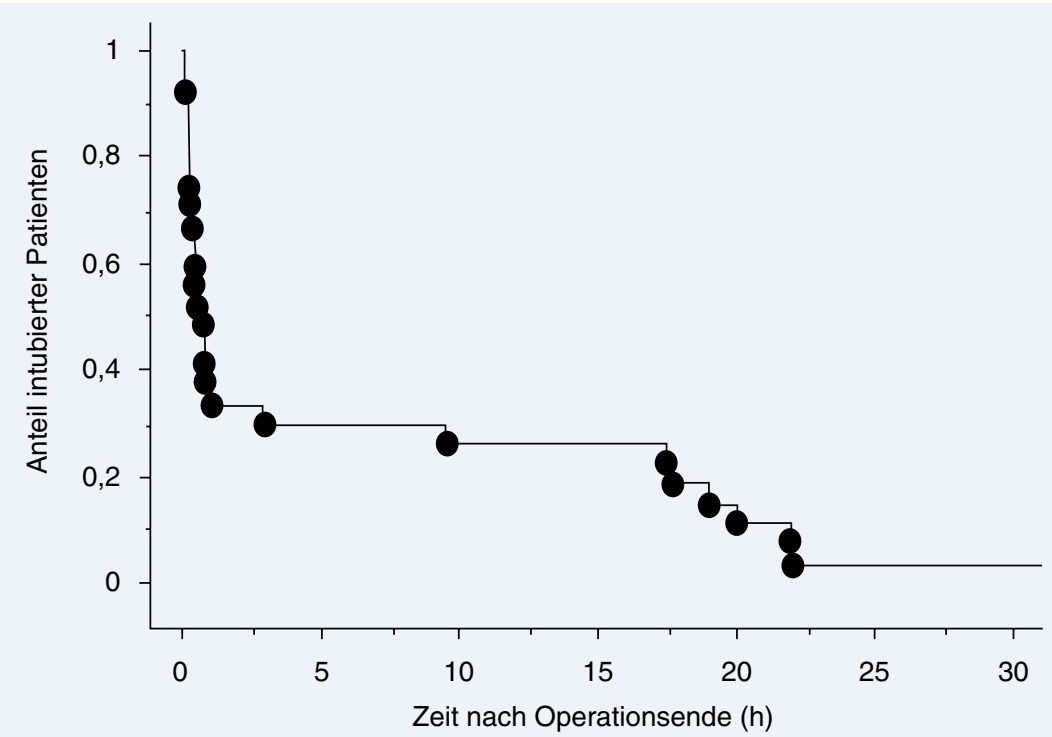

Abb. $2 \Delta$ Mayer-Kaplan-Plot: Anteil noch intubierter Kinder nach Operationsende ( $n=28$ Patienten)

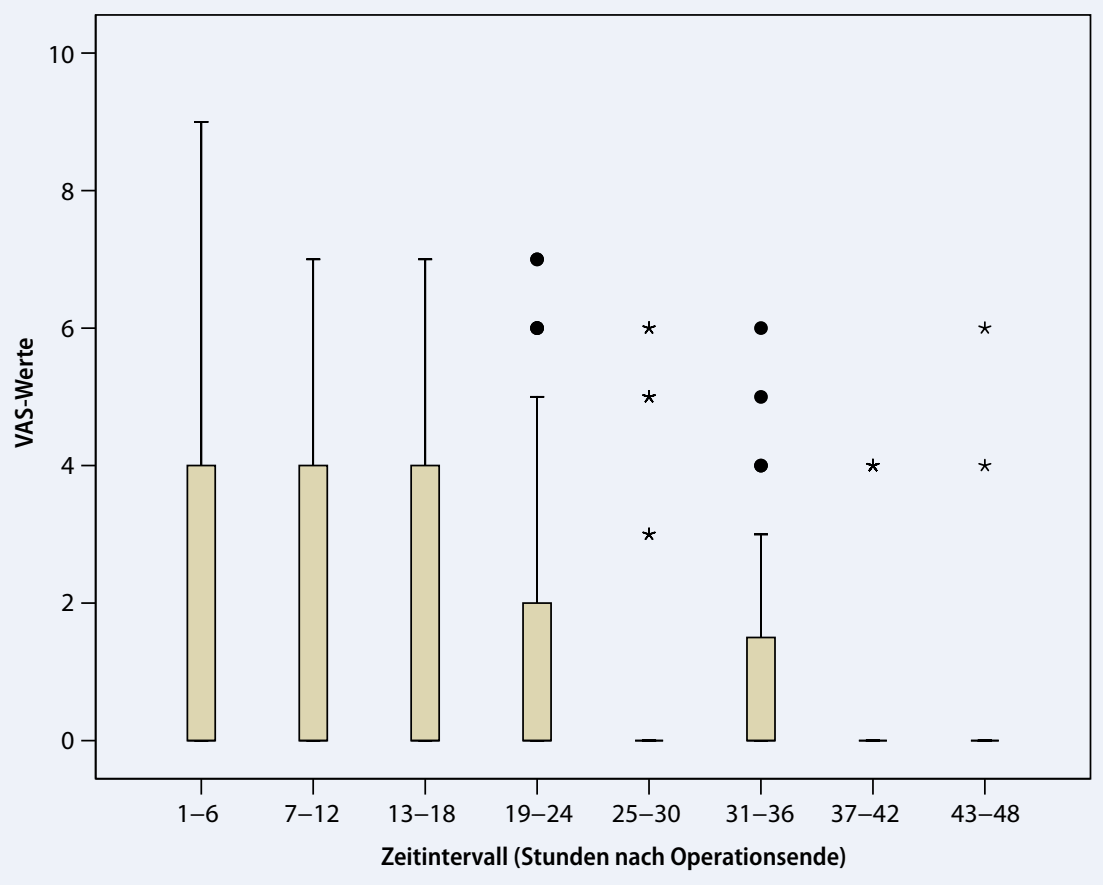

Abb. $3 \Delta$ Verlauf der VAS-Scores in den ersten $48 \mathrm{~h}$ postoperativ ( $\mathrm{n}=28$ Patienten, insgesamt 332 VASScores zu verschiedenen Zeitpunkten). Die Boxplots fassen jeweils die VAS-Scores aller Patienten in einem 6-h-Intervall zusammen. Die Mediane fallen auf einen VAS $=0$

Eröffnung der Dura mater bei den $6 \mathrm{~Pa}$ tienten mit intrathekaler Opioidinjektion durch den Chirurgen wurde mit 5,4 $\mu \mathrm{g} /$ kgKG Fentanyl (ein Patient) oder 50,6 $\mu \mathrm{g} /$ $\mathrm{kgKG}(35,7-69,4 \mu \mathrm{g} / \mathrm{kgKG})$ Alfentanil i.v. gewährleistet. Von den 22 Patienten mit erfolgreicher transkutaner Lumbalpunktion erhielten 12 Patienten $15,4 \mu \mathrm{g} / \mathrm{kgKG}$ $(6,6-37, \mathrm{o} \mu \mathrm{g} / \mathrm{kgKG})$ Alfentanil und ein Pa-
Die Anästhesie wurde mithilfe niedriger Konzentrationen von Sevofluran (23 Patienten, o,8-1,2 Vol.-\%, Median 1,2 Vol.-\%) oder kontinuierlicher Propofolinfusion (5 Patienten, $5-11 \mathrm{mg} / \mathrm{kgKG}$ und h, Median $7 \mathrm{mg} / \mathrm{kgKG}$ und h) aufrechterhalten; die Beatmung erfolgte mit einem Gemisch von 50\%igem Lachgas in Sauerstoff. Der chirurgische Eingriff dauerte im Mittel $258 \mathrm{~min}(8 \mathrm{o}-485 \mathrm{~min})$. Die Extubation erfolgte bei 17 Patienten bereits im OP während eines Zeitraums von 10-70 min nach Beendigung des Eingriffs (- Abb. 2). Aufgrund einer Obstruktion der oberen Atemwege benötigten 2 Patienten einen nasopharyngealen (Wendl-) Tubus. Der Entschluss zu einer verzögerten Extubation auf der Intensivpflegestation wurde bei 11 Patienten getroffen; dabei lag bei 3 Patienten eine Massentransfusion, bei 4 Patienten eine schwere vorbestehende Muskelschwäche und bei einem weiteren eine Hypothermie $<35^{\circ} \mathrm{C}$ vor. $\mathrm{Zu}$ dem bestand in einem Fall eine Hypoplasie der linken Lunge und der linken Lungenarterie im Rahmen einer schweren Thoraxdeformität, bei 2 weiteren Patienten bestanden Dysmorphiesyndrome mit zusätzlicher Trachealstenose in einem sowie mit intermittierendem Vorhofflattern bei Mitralinsuffizienz im anderen Fall. Es wurden 3 von 11 Kindern am selben Tag und 7 am nächsten Morgen extubiert. Bis auf einen einzigen Patienten, der auch auf der Intensivpflegestation fortwährend massive Transfusionen benötigte, waren alle Patienten nach spätestens $24 \mathrm{~h}$ extubiert worden.

Innerhalb der ersten $48 \mathrm{~h}$ postoperativ wurden im Mittel o,1 mg/kgKG und h (o-o,2 $\mathrm{mg} / \mathrm{kgKG}$ und h) Nalbuphin kontinuierlich infundiert. Die Gesamtdosis der Nalbuphinboli während dieses Zeitraums betrug im Mittel o,71 mg/kgKG in $48 \mathrm{~h}(\mathrm{o}-3,44 \mathrm{mg} / \mathrm{kgKG}$ in $48 \mathrm{~h}$; arithmetisches Mittel 1,26 mg/kgKG in $48 \mathrm{~h}$ ). Zwei Patienten erhielten bei völlig unauffälligem, schmerzfreiem postoperativen Verlauf kein Nalbuphin. Zur Beurteilung der postoperativen Analgesie wurden in den ersten $48 \mathrm{~h}$ nach Beendigung der Operation insgesamt 332 VASScores erhoben. Der Verlauf der VASScores während dieses Zeitraums ist in - Abb. 3 dargestellt. Durch die Kombination von intrathekal verabreichten 


\section{A. Schmitz · B. Salgo · M. Weiss · C.M. Dillier · A. Frotzler · A.C. Gerber Intrathekale Opioidmedikation zur perioperativen Analgesie bei schwer behinderten Kindern mit Wirbelsäulenoperationen}

\section{Zusammenfassung}

Hintergrund. An gesunden Kindern konnte gezeigt werden, dass intrathekal verabreichte Opioide eine sichere und effektive perioperative Analgesie bei wirbelsäulenchirurgischen Eingriffen ermöglichen. Das Ziel der vorliegenden Untersuchung ist es, ihre Anwendbarkeit bei schwer- und schwerstbehinderten Kindern für wirbelsäulenchirurgische Eingriffe zu überprüfen.

Methode. Mit Zustimmung der lokalen Ethikkommission wurden Patienten vom Status 3 und 4 der Klassifikation der American Society of Anesthesiologists (ASA), die sich einem wirbelsäulenchirurgischen Eingriff unterziehen mussten, retrospektiv untersucht. Zusätzlich zur Allgemeinanästhesie mit Sevofluran oder i.v.-verabreichtem Propofol erhielten die Patienten vor der Operation $20 \mu \mathrm{g} / \mathrm{kgKG}$ Morphin und 1,5 $\mu \mathrm{g} / \mathrm{kgKG}$ Sufentanil intrathekal. Nach Beendigung des Eingriffs wurde eine Nalbuphindauerinfusi- on gestartet. Der Bedarf an zusätzlichen intra- und postoperativen Analgetika, der Zeitpunkt der Extubation, die postoperativen Schmerz-Scores und die arteriellen Kohlendioxidpartialdruck- $\left(\mathrm{p}_{\mathrm{a}} \mathrm{CO}_{2}\right)$-Werte sowie das Auftreten unerwünschter Nebenwirkungen wurden untersucht.

Ergebnisse. Es wurden 28 Patienten im Alter von 2,8 bis 18,5 Jahren (Median 11,6 Jahre) untersucht. Unmittelbar im OP konnten 17 Patienten extubiert werden; bei 11 Patienten entschied man sich zu einer verzögerten Extubation. Bis auf einen Patienten mit postoperativer Massivtransfusion konnten alle innerhalb von $24 \mathrm{~h}$ extubiert werden. Eine adäquate postoperative Analgesie mit SchmerzScores $\leq 3$ wurde mithilfe der Kombination von intrathekal verabreichten Opioiden mit Nalbuphin postoperativ bei 26 von $28 \mathrm{~Pa}$ tienten (93\%) erreicht. Bei 2 Patienten war der Wechsel auf eine i.v.-Morphin-Gabe er- forderlich. Postoperative Übelkeit und Erbrechen ("postoperative nausea and vomiting“, PONV), Pruritus und leichte Hypoventilation mit $\mathrm{p}_{\mathrm{a}} \mathrm{CO}_{2}$-Werten zwischen 5,2 bis $9,7 \mathrm{kPa}$ (Median 6,3 kPa) waren die beobachteten Nebenwirkungen.

Schlussfolgerung. Der Einsatz intrathekal verabreichter Opioide, ergänzt durch eine postoperative Nalbuphininfusion, erlaubt in den allermeisten Fällen eine frühzeitige Extubation ohne persistierende Atemdepression und ist eine praktikable sowie effektive Methode zur postoperativen Analgesie bei schwer behinderten Kindern nach wirbelsäulenchirurgischen Eingriffen.

Schlüsselwörter

Opioide $\cdot$ Intrathekal $\cdot$ Wirbelsäulenchirurgie $\cdot$ Kinder

\section{Intrathecal opioid medication for perioperative analgesia in severely handicapped children undergoing spinal operations}

\section{Abstract}

Purpose of the study. Intrathecal opioids have been shown to be safe and effective for postoperative analgesia in healthy children for spinal surgery. The aim of this study was to evaluate the applicability of intrathecal opioids in severely handicapped children scheduled for spinal surgery.

Methods. With hospital ethical committee approval, patients with physical states III and IV of the ASA classification requiring spinal surgery were retrospectively studied. In addition to inhalational anesthesia with sevoflurane or intravenous anesthesia using propofol, morphine $20 \mu \mathrm{g} / \mathrm{kgBW}$ and sufentanil $1.5 \mu \mathrm{g} / \mathrm{kgBW}$ were administered intrathecally before surgery. After surgery an infusion of nalbuphine was started. Need for additional intraoperative and postoperative analgesics, time of extubation, postoperative pain scores and $p_{\mathrm{a}} \mathrm{CO}_{2}$ values as well as adverse effects were recorded.

Results. A total of 28 patients aged from 2.8 to 18.5 years (median 11.6 years) were studied. Immediate tracheal extubation in the operating room was possible in 17 patients and for 11 patients delayed extubation was elected. All patients were extubated within $24 \mathrm{~h}$ except for 1 patient who received massive postoperative transfusions. In 26 out of 28 patients $(93 \%)$ the combination of intrathecal opioids with postoperative nalbuphine provided adequate analgesia. Observed side ef- fects were post-operative nausea and vomiting (PONV), pruritus and moderate hypoventilation. In two patients a change to intravenous morphine therapy was necessary. Conclusion. The use of intrathecal opioids for perioperative pain control from spinal fusion in severely handicapped children is feasible. Intrathecal opioids provide adequate postoperative analgesia and allow early extubation without persisting relevant respiratory compromise in most of these patients.

Keywords

Opioids · Intrathecal $\cdot$ Spinal surgery .

Children 


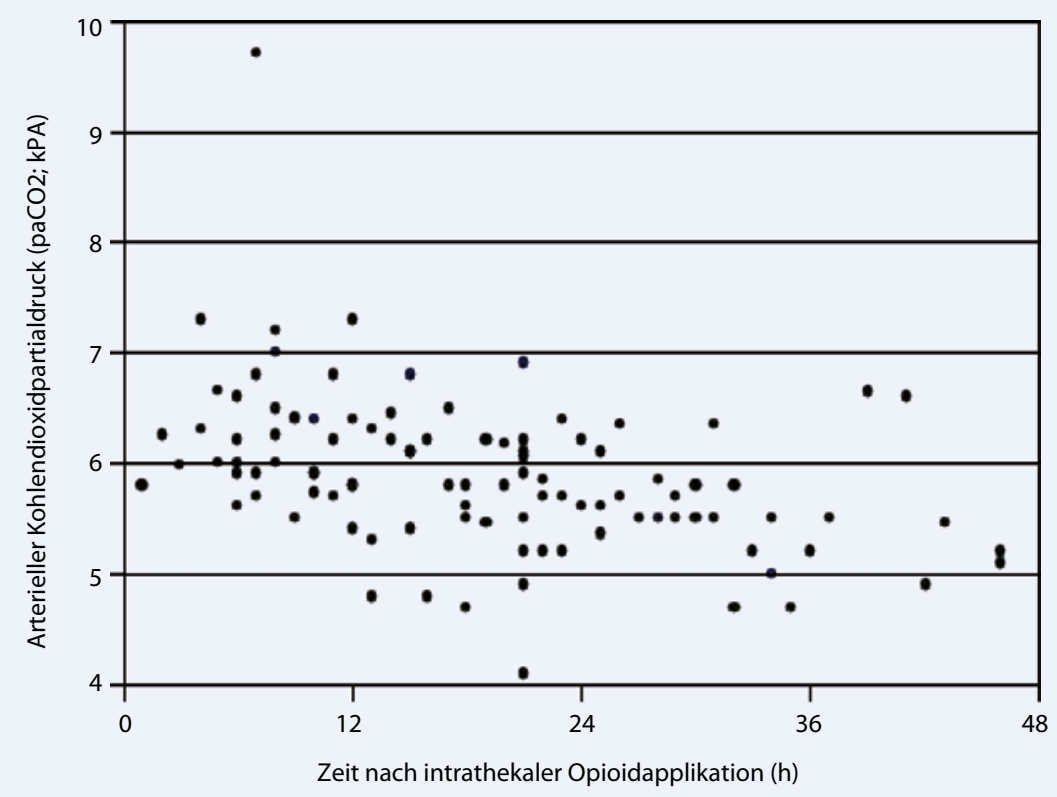

Abb. $4 \Delta \mathrm{p}_{\mathrm{a}} \mathrm{CO}_{2}$-Werte nach intrathekaler Opioidgabe ( $\mathrm{n}=27$ Patienten)

Opioiden, Paracetamol, Nalbuphindauerinfusion und bei Bedarf applizierten Nalbuphinboli konnte bei 26 von $28 \mathrm{~Pa}$ tienten (93\%) eine mit einem VAS-Score $\leq_{3}$ als adäquat beurteilte Analgesie erreicht werden. Bei 2 von 28 Patienten, von denen einer noch im $\mathrm{OP}$ und einer verzögert extubiert wurde, wurde durch diese Medikation keine adäquate Analgesie erreicht. Die Analgesie wurde bei beiden Patienten nach einem initialen Morphinbolus auf eine kontinuierliche i.v.Morphin-Infusion gewechselt.

Der mediane $\mathrm{p}_{\mathrm{a}} \mathrm{CO}_{2}$-Wert in den ersten $24 \mathrm{~h}$ bei den extubierten Patienten war $6,3 \mathrm{kPa}(5,2-9,7 \mathrm{kPa})$. Ein deutlich erhöhter initialer $\mathrm{p}_{\mathrm{a}} \mathrm{CO}_{2}$ von $9,7 \mathrm{kPa}$ bei einem Patienten unmittelbar nach Extubation im OP verbesserte sich nach Einlage eines Wendl-Tubus innerhalb $1 \mathrm{~h}$ auf $7,2 \mathrm{kPa}$. Die Atemfrequenz betrug dabei $13 / \mathrm{min}$ bzw. nach $1 \mathrm{~h} 10 / \mathrm{min}$. Bis zu $12 \mathrm{~h}$ nach der intrathekalen Opioidinjektion wurden $\mathrm{p}_{\mathrm{a}} \mathrm{CO}_{2}$-Werte über $7 \mathrm{kPa}$ gemessen, nicht jedoch zu einem späteren Zeitpunkt (• Abb. 4). Es wurde bei keinem Patienten eine Naloxongabe oder eine Reintubation erforderlich. Postoperatives Erbrechen und Übelkeit traten bei 11 Patienten (39\%) auf. Pruritus wurde bei 2 Patienten $(7 \%)$ beobachtet. Weitere unerwünschte Ereignisse gab es nicht.

Die Verlegung von der Intensivpflegestation auf die Normalabteilung konn- te bei 17 von 28 Patienten innerhalb von $24 \mathrm{~h}$, bei weiteren 8 Patienten innerhalb von $48 \mathrm{~h}$ erfolgen. Lediglich 3 Patienten wurden länger als $48 \mathrm{~h}$ auf der Intensivpflegestation behandelt; die Ursache war bei allen 3 Patienten eine relevante postoperative Blutung und Transfusionstherapie.

\section{Diskussion}

\section{Ziele der Anästhesieführung}

Stabile intraoperative Verhältnisse, frühe Extubation und gute postoperative Analgesie sind die wesentlichen Ziele der Anästhesieführung bei wirbelsäulenchirurgischen Eingriffe an körperlich und/ oder mental behinderten Kindern. Gegenüber gesunden Kindern bestehen häufig verschiedene zusätzliche Risikofaktoren. Hierzu gehören insbesondere ein erhöhter intraoperativer Blutverlust infolge thrombozytärer oder vaskulärer Dysfunktion [4, 11] sowie stark eingeschränkte respiratorische Reserven bei einer restriktiven Ventilationsstörung, Muskelschwäche oder Status nach wiederholten Aspirationen $[17,22]$. Zusätzlich besteht die Gefahr einer suboptimalen postoperativen Analgesie, da viele dieser Kinder nicht in der Lage sind, ihre Schmerzen adäquat auszudrücken oder eine Schmerzpumpe selbstständig zu bedienen [15].

\section{Intrathekal verabreichte Opioide}

Verschiedene Autorengruppen konnten zeigen, dass intrathekal verabreichte Opioide intraoperativ einen stabilen hämodynamischen Verlauf ermöglichen, den Blutverlust reduzieren $[3,5,6,9]$ und dabei für viele Patienten eine hervorragende postoperative Analgesie über $24-48 \mathrm{~h}$ ermöglichen, ohne dass eine Kooperation oder Eigenaktivität der Kinder hierfür erforderlich gewesen wäre $[3,5,6,8,20]$. Jedoch treten häufig Nebenwirkungen wie frühe und späte Atemdepression [1], PONV und Pruritus auf $[5,6,8,20]$. In den meisten Studien führen höhere Opioiddosen zu einer besseren und länger anhaltenden Analgesie, jedoch besteht weder für die Analgesie noch für Nebenwirkungen eine lineare Dosis-Wirkung-Beziehung [5, 6]. Bei gynäkologischen Patientinnen wurden bereits verschiedene Studien mit epiduraler Opiatanalgesie durchgeführt, um bei erhaltener analgetischer Wirkung eine Reduktion bzw. Abschwächung der Nebenwirkungen PONV oder Pruritus mithilfe von Naloxon oder Nalbuphin in niedriger oder sehr niedriger Dosis zu bewirken. Einige der Arbeiten mit Nalbuphin führten zu widersprüchlichen Ergebnissen [12, 13, 16], andere konnten wiederum den gewünschten Effekt nachweisen [2, 21]. Aufgrund dieser Ergebnisse fiel die Entscheidung bei den hier vorgestellten Patienten für eine hochdosierte intrathekale Opioiddosis, kombiniert mit einer niedrig dosierten postoperativen Nalbuphindauerinfusion. Die theoretische Überlegung, dass Nalbuphin als gemischter Agonistantagonist am $\mu$-Rezeptor eine Abschwächung der Nebenwirkungen Atemdepression, PONV und Pruritus und via $\kappa$-Rezeptoren zudem eine gewisse Analgesie bewirkt, unterstützt dieses Vorgehen. Für die intrathekale Opioidapplikation wurde eine Kombination von Sufentanil und Morphin gewählt, um einerseits intraoperativ eine hohe Opioidkonzentration und andererseits eine lange Wirkungspersistenz bis $\mathrm{zu} 48 \mathrm{~h}$ postoperativ zu erreichen [3].

Mit dieser Arbeit konnte gezeigt werden, dass die perioperative Analgesie mit intrathekal applizierten $20 \mu \mathrm{g} / \mathrm{kg}$ KG Morphin und 1,5 $\mu \mathrm{g} / \mathrm{kgKG}$ Sufenta- 
nil, ergänzt durch eine postoperative i.v.Nalbuphin-Dauerinfusion $(0,1 \mathrm{mg} / \mathrm{kg}$ KG und $h$ ) bei schwer behinderten Kindern nach wirbelsäulenchirurgischen Eingriffen zu einer effektiven postoperativen Analgesie führte und bei den meisten dieser Patienten eine frühzeitige Extubation ohne relevante respiratorische Beeinträchtigungen ermöglichte. Außer bei einem Patienten gelang die Extubation entweder unmittelbar im OP nach Beendigung des Eingriffs oder spätestens innerhalb $24 \mathrm{~h}$ postoperativ. Hypoventilation war kein klinisch relevantes Problem, obwohl viele der Patienten bereits präoperativ über nur eingeschränkte respiratorische Reserven verfügten. Nach der Extubation zeigte sich zwar regelmäßig eine leichte Hyperkapnie, ohne dass jedoch eine Reintubation oder Naloxongabe erforderlich gewesen wäre. Der initial hohe $\mathrm{p}_{\mathrm{a}} \mathrm{CO}_{2}$-Wert von $9,7 \mathrm{kPa}$ bei einem Patienten war v. a. durch eine obstruktive Komponente der oberen Luftwege bedingt und besserte sich nach Einlage eines nasopharyngealen Atemwegs.

Die Kombination der hochdosierten intrathekal verabreichten Opioide mit der moderat dosierten postoperativen Nalbuphindauerinfusion, bei Bedarf ergänzt durch Nalbuphinboli, sowie einer Basisanalgesie mit Paracetamol führte bei 93\% der Patienten zu einer adäquaten Analgesie. Eine Eskalation der Schmerztherapie mit i.v.-Morphin-Gabe war lediglich bei 2 Patienten erforderlich. Bei diesem Patientengut wurde kein Neuromonitoring durchgeführt, obwohl intrathekal verabreichte Opioide hiermit nicht interferieren [8].

Dank der erfolgreichen Analgesietechnik und des Fehlens relevanter Episoden einer Atemdepression konnten $60 \%$ der Patienten bereits am Morgen des ersten postoperativen Tages von der Intensiv- auf die Normalstation wechseln; insgesamt konnten $90 \%$ der Patienten innerhalb von maximal $48 \mathrm{~h}$ nach Aufnahme von der Intensivstation verlegt werden.

Die hohe PONV-Inzidenz ist ein unbefriedigendes Resultat dieser Studie. Als Konsequenz ergibt sich die Empfehlung $\mathrm{zu}$ einer erweiterten antiemetischen Prophylaxe, z. B. mit Dexamethason nach Anästhesieeinleitung [10].

\section{Limitierungen der Studie}

Die vorliegende Beobachtungsstudie hat einige offensichtliche Einschränkungen. Aufgrund des Fehlens einer Kontrollgruppe können die Ergebnisse nur mit solchen weniger stark behinderter Kinder verglichen werden. Obwohl in den meisten Fällen - schon aufgrund einer eingeschränkten Kooperationsfähigkeit - keine präoperativen Lungenfunktionsteste durchgeführt wurden, könnten die Patienten am ehesten mit denjenigen von Rawlins et al. [17] sowie Wazeka et al. [22] verglichen werden, die pädiatrische Patienten mit schwerer restriktiver Lungenerkrankung untersucht haben. Hinsichtlich pulmonaler Komplikation sind die hier vorliegenden Ergebnisse äußerst positiv zu werten, da bei keinem der Patienten eine Reintubation, Tracheostomie, Langzeittherapie mit Sauerstoff oder Langzeitbeatmung durchgeführt wurde. Kein einziger Patient benötigte Naloxon zur Antagonisierung einer opiatinduzierten Atemdepression.

Die Schmerzerfassung bei den Patienten mit teilweise eingeschränkter Kooperations- und Kommunikationsfähigkeit ist nicht unproblematisch und zu einem Großteil von der Fremdeinschätzung durch Pflegende und Eltern abhängig; dennoch ist ein Vorteil in einer Quantifizierung mithilfe der VAS gegeben.

In dieser Studie wurde nicht die Frage untersucht, ob die intrathekale Opioidapplikation in der Gruppe der schwer behinderten Kinder effektiver in der postoperativen Analgesie ist als eine systemische postoperative Analgesie. Während die erfolgreiche intraoperative Analgesiewirkung der intrathekal verabreichten Opioide offensichtlich ist, kann aufgrund des multimodalen Ansatzes mit zusätzlicher Paracetamol- und Nalbuphinapplikation der Anteil der einzelnen Komponenten am postoperativen Erfolg nicht beurteilt werden. Bei den überwiegend sehr tiefen Schmerz-Scores könnte bei reiner Betrachtung der Analgesie eingewendet werden, dass möglicherweise nicht alle Substanzen erforderlich waren - immerhin erhielten 2 Patienten tatsächlich kein Nalbuphin. Zuverlässige Aussagen zur Sicherheit der eingesetzten Methode sind aufgrund der relativ geringen Patientenzahl ebenfalls nicht möglich.

\section{Schlussfolgerung}

Die Studie zeigt, dass intrathekal verabreichte Opioide bei schwer behinderten Kindern, die sich einem wirbelsäulenchirurgischen Eingriff unterziehen müssen, einen reibungslosen postoperativen Verlauf mit rascher Verlegungsfähigkeit von der Intensivpflegestation ermöglichen. In der Praxis sieht sich der Anästhesist ansonsten durch den Einsatz systemischer Opiate häufig einem Balanceakt zwischen suffizienter Analgesie und noch erhaltener Spontanatmung ausgesetzt.

\section{Fazit für die Praxis}

Die intrathekale Applikation von $20 \mu \mathrm{g} /$ kgKG Morphin und 1,5 $\mu \mathrm{g} / \mathrm{kgKG}$ Sufentanil, ergänzt durch eine postoperative Nalbuphindauerinfusion, erlaubt in den allermeisten Fällen die frühzeitige Extubation ohne relevante persistierende Atemdepression und ist eine praktikable sowie effektive Methode zur postoperativen Analgesie bei schwer behinderten Kindern nach wirbelsäulenchirurgischen Eingriffen.

\section{Korrespondenzadresse \\ Dr. A. Schmitz}

Anästhesieabteilung, Universitäts-Kinder-

kliniken Zürich

Steinwiesstrasse 75, 8032 Zürich

Schweiz

achim.schmitz@kispi.uzh.ch

Interessenkonflikt. Der korrespondierende Autor gibt an, dass kein Interessenkonflikt besteht.

\section{Literatur}

1. Blackman RG, Reynolds J, Shively J (1991) Intrathecal morphine: dosage and efficacy in younger patients for control of postoperative pain following spinal fusion. Orthopedics 14:555-557

2. Cohen SE, Ratner EF, Kreitzman TR et al (1992) Nalbuphine is better than naloxone for treatment of side effects after epidural morphine. Anesth Analg 75:747-752

3. Dalens B, Tanguy A (1988) Intrathecal morphine for spinal fusion in children. Spine (Phila Pa 1976) 13:494-498

4. Edler A, Murray DJ, Forbes RB (2003) Blood loss during posterior spinal fusion surgery in patients with neuromuscular disease: is there an increased risk? Paediatr Anaesth 13:818-822 
5. Eschertzhuber $\mathrm{S}$, Hohlrieder $\mathrm{M}$, Keller $\mathrm{C}$ et al (2008) Comparison of high- and low-dose intrathecal morphine for spinal fusion in children. Br J Anaesth 100:538-543

6. Gall O, Aubineau JV, Berniere J et al (2001) Analgesic effect of low-dose intrathecal morphine after spinal fusion in children. Anesthesiology 94:447452

7. Ganesh A, Kim A, Casale P et al (2007) Low-dose intrathecal morphine for postoperative analgesia in children. Anesth Analg 104:271-276

8. Gibson PR (2004) Anaesthesia for correction of scoliosis in children. Anaesth Intensive Care 32:548-559

9. Goodarzi M (1998) The advantages of intrathecal opioids for spinal fusion in children. Paediatr Anaesth 8:131-134

10. Holt R, Rask P, Coulthard KP et al (2000) Tropisetron plus dexamethasone is more effective than tropisetron alone for the prevention of postoperative nausea and vomiting in children undergoing tonsillectomy. Paediatr Anaesth 10:181-188

11. Kannan S, Meert Kl, Mooney JF et al (2002) Bleeding and coagulation changes during spinal fusion surgery: a comparison of neuromuscular and idiopathic scoliosis patients. Pediatr Crit Care Med 3:364-369

12. Kendrick WD, Woods AM, Daly MY et al (1996) Naloxone versus nalbuphine infusion for prophylaxis of epidural morphine-induced pruritus. Anesth Analg 82:641-647

13. Kjellberg F, Tramer MR (2001) Pharmacological control of opioid-induced pruritus: a quantitative systematic review of randomized trials. Eur J Anaesthesiol 18:346-357

14. Krechel SW, Helikson MA, Kittle D et al (1995) Intrathecal morphine (ITM) for postoperative pain control in children: a comparison with nalbuphine patient controlled analgesia (PCA). Paediatr Anaesth 5:177-183

15. Malviya S, Voepel-Lewis T, Tait AR et al (2001) Pain management in children with and without cognitive impairment following spine fusion surgery. Paediatr Anaesth 11:453-458

16. Nakatsuka N, Minogue SC, Lim J et al (2006) Intravenous nalbuphine 50 microg $\times \mathrm{kg}(-1)$ is ineffective for opioid-induced pruritus in pediatrics. Can J Anaesth 53:1103-1110

17. Rawlins BA, Winter RB, Lonstein JE et al (1996) Reconstructive spine surgery in pediatric patients with major loss in vital capacity. J Pediatr Orthop 16:284-292

18. Suominen PK, Ragg PG, Mckinley DF et al (2004) Intrathecal morphine provides effective and safe analgesia in children after cardiac surgery. Acta Anaesthesiol Scand 48:875-882

19. Theroux MC, Akins RE (2005) Surgery and anesthesia for children who have cerebral palsy. Anesthesiol Clin North America 23:733-743

20. Tobias JD (2004) A review of intrathecal and epidural analgesia after spinal surgery in children. Anesth Analg 98:956-965

21. Wang JJ, Ho ST, Tzeng JI (1998) Comparison of intravenous nalbuphine infusion versus naloxone in the prevention of epidural morphine-related side effects. Reg Anesth Pain Med 23:479-484

22. Wazeka AN, Dimaio MF, Boachie-Adjei O (2004) Outcome of pediatric patients with severe restrictive lung disease following reconstructive spine surgery. Spine (Phila Pa 1976) 29:528-534; discussion 535
„Der Anaesthesist“ bietet Ihnen jeden Monat umfassende und aktuelle Beiträge zu interessanten Themenschwerpunkten zu allen Aspekten der Anästhesie und Intensivmedizin bis hin zur Schmerztherapie.

Wir haben die Jahrgänge 2009/2010 im Überblick für Sie zusammengestellt:

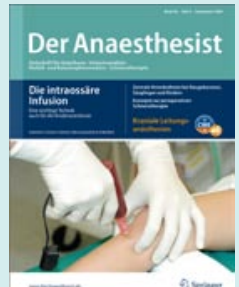

2009

- 01/2009 Opioidinduzierte Immunsuppression

- 02/2009 Kortikosteroidinsuffizienz bei kritisch Kranken

03/2009 Palliativmedizin

- 04/2009 Pathomechanismen des Organversagens

- 05/2009 Zur Delegation ärztlicher Leistungen

- 06/2009 Die familienfreundliche Klinik

- 07/2009 Perioperative Myokardschäden

- 08/2009 Hämodynamisches Monitoring in der perioperativen Phase

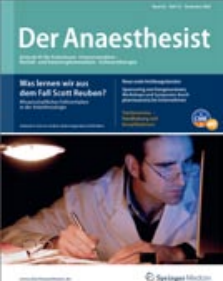

- 09/2009 Die intraossäre Infusion

- 10/2009 Patienten mit Koronarstents

- 11/2009 Hämodynamisches Monitoring bei Einlungenventilation

12/2009 Was lernen wir aus dem Fall Scott Reuben?
2010

- 01/2010

- 02/2010

- 03/2010

- 04/2010

- 05/2010

$-06 / 2010$

- 07/2010
Neue pandemische (A)H1N1-Influenza

Patientenverfügung

Kommunikation mit ängstlichen Patienten

Perioperatives Management bei Zeugen Jehovas Suizidalität, persönliche Krisen und Burnout bei Ärzten Implantation von Cardioverter-Defibrillatoren Protektive Beatmungstherapie
Möchten Sie ein bereits erschienenes Heft nachbestellen? Dieses können Sie direkt bei unserem Kundenservice zum Preis von je EUR 33,- beziehen.

\section{So erreichen Sie unseren Kundenservice:}

Springer Customer Service Center GmbH

Haberstr. 7

69126 Heidelberg

Tel.: +49 $6221345-4303$

Fax: +496221345-4229

E-Mail: Leserservice@springer.com

\section{Beiträge auf einen Blick}

Abonnenten haben online Zugriff auf alle Beiträge im elektronischen Volltextarchiv unter

\section{www.DerAnaesthesist.de}

Ins Volltextarchiv gelangen Sie von der Startseite über den Navigationspunkt "Online-Version".

Eine interessante Lektüre wünscht Ihnen

Ihre Redaktion

Fachzeitschriften Medizin/Psychologie 